\section{MOLECULAR INSIGHTS ON SAFETY AND ANTI-TUMOR ACTIVITY OF A NON-IRAE-INDUCING ANTI-CTLA-4 MONOCLONAL ANTIBODY ONC-392}

${ }^{1}$ Yang Liu*, ${ }^{2}$ Yan Zhang, ${ }^{3}$ Xuexiang Du, ${ }^{3}$ Mingyue Liu, ${ }^{4}$ Xianfeng Fang, ${ }^{4}$ Libing Mu, ${ }^{1}$ Vadim Tevetnitsky, ${ }^{1}$ Martin Devenport, ${ }^{1}$ Pan Zheng. ${ }^{1}$ OncoC4, Inc., Rockville, MD, United States; ${ }^{2}$ Shanghai Institute of Immunology, Shanghai, China; ${ }^{3}$ University of Maryland Baltimore, Jinan, China; ${ }^{4}$ Acrolmmune, Ltd, Guangzhou, China

Background Anti-CTLA-4 antibodies have brought about limited clinical benefit because severe toxicity limits dosing levels and/or duration. We used CTLA-4 knockin mice to screen for antibodies with higher anti-tumor activity but lower autoimmunity. We have revealed that the key for better safety and preclinical efficacy is preservation of CTLA-4 for immune tolerance and intratumorial Treg depletion. Our work established that, independent of blocking activities, mAbs that preserve CTLA-4 recycling maintain the physiological immune tolerance checkpoint function while allowing more efficient and selective elimination of tumor-infiltrating regulatory $\mathrm{T}$ cells, resulting in highest efficacy and lowest toxicity and was thus developed for clinical testing of all antibodies tested. ${ }^{1-6}$ The antibody with best safety and efficacy profile, ONC-392 was developed for clinical testing. The first-in human studies showed that ONC-392 is safe and well tolerated. Remarkably, no irAE has been reported among patients who has received repeated dosing of $3.0 \mathrm{mg} / \mathrm{kg}$ and $10.0 \mathrm{mg} / \mathrm{kg}$ of ONC-392. The molecular and cellular characterization of ONC-392 will be presented.

Methods In vitro binding and disassociation assay were determined between $\mathrm{pH}$ 4.0-7.0. The intracellular traffic of both antibodies and CTLA- 4 molecules were visualized by confocal microscopy. The binding to human and mouse FcgRI, IIA, IIB, and III (A), FcRn as well as mouse FcgRIV were evaluated by surface plasmon resonance (SPR). Depletion of regulatory $\mathrm{T}$ cells in tumor and lymphoid tissues were determined by flow cytometry.

Results ONC-392 is a pH-sensitive antibody that preserves CTLA-4 recycling. By preserving cell surface CTLA-4, Onco392 preserves immune tolerance. Preserving CTLA-4 on tumor-infiltrating Treg contribute to more effective immunotherapy. In addition to its unique feature of $\mathrm{pH}$ sensitive binding, OncoC4 also have several important features in Fc. ONC-392 shown comparable binding to human FcgRI and IIIA as wild-type IgG1s. As expected from the mutations introduced, ONC-392 show about 6 fold higher affinity for $\mathrm{FcRn}$ than wild-type IgG1. Interestingly, ONC-392 has shown 7-10-fold reduction to FcgRIIB, which is generally considered to be a negative signaling FcR. ONC-392 binding to mouse FcgRI-IV was lower that WT IgG1.

Conclusions Unlike other clinical anti-CTLA-4 antibodies, ONC-392 preserves CTLA-4 recycling and thus Treg function in the peripheral tissues. The higher cell surface CTLA-4 allows more efficient Treg depletion in the tumor microenvironment. In addition, despite reduced binding to mouse activating Fc?RI, III/IV, ONC-392 was more effective in intratumor Treg depletion in the mice. Therefore, lacking negative signaling from Fc?RIIB may also contribute to its anti-tumor activity.

Trial Registration NCT04140526

\section{REFERENCES}

1. Du $X$, et al. Uncoupling therapeutic from immunotherapy-related adverse effects for safer andeffective anti-CTLA-4 antibodies in CTLA4 humanized mice. Cell Res 2018:28:433-447.
2. Du $X$, et al. A reappraisal of CTLA-4 checkpoint blockade in cancer immunotherapy. Cell Res 2018;28:416-432.

3. Liu Y, Zheng P. How does an anti-CTLA-4 antibody promote cancer immunity? Trends Immunol 2018:39:953-956.

4. Zhang $Y$, et al. Hijacking antibody-induced CTLA-4 lysosomal degradation for safer and more effective cancer immunotherapy. Cell Res 2019;29:609-627.

5. Liu Y, Zheng P. Preserving the CTLA-4 checkpoint for safer and more effective cancer immunotherapy. Trends Pharmacol Sci 2020;41(1):4-12.

6. Zhang $\mathrm{P}$, et al. Mechanism- and immune landscape-based ranking of therapeutic responsiveness of 22 major human cancers to next generation anti-CTLA-4 antibodies. Cancers 2020;12:284.

http://dx.doi.org/10.1136/jitc-2021-SITC2021.231 